\title{
Reply to comments on "Robotic-assisted right medial and anterior basal segmentectomy (S7+S8)": lymph node dissection and incision
}

\author{
Jian-Tao $\mathrm{Li}^{1,2}$, Qing-Quan Luo ${ }^{1,2}$ \\ ${ }^{1}$ Shanghai Lung Tumor Clinical Medical Center, Shanghai Chest Hospital, Shanghai Jiao Tong University, Shanghai 200030, China; ${ }^{2}$ School of \\ Medicine, Jiangsu University, Zhenjiang 212013, China \\ Correspondence to: Qing-Quan Luo. Shanghai Lung Tumor Clinical Medical Center, Shanghai Chest Hospital, Shanghai Jiao Tong University, \\ Shanghai 200030, China; School of Medicine, Jiangsu University, Zhenjiang 212013, China. Email: qingquan_luo@hotmail.com. \\ Response to: Hanna WC. Uncharted territory. J Thorac Dis 2019;11:628-9. \\ Kim DY, Jeong JY. Robotic surgery for pulmonary segmentectomy. J Thorac Dis 2019;11:624-7.
}

Submitted Sep 03, 2019. Accepted for publication Sep 25, 2019.

doi: $10.21037 /$ jtd.2019.09.74

View this article at: http://dx.doi.org/10.21037/jtd.2019.09.74

In this article, we respond to the comments made by Dr. Hanna, Drs. Kim and Jeong on "Robotic-assisted right medial and anterior basal segmentectomy $(\mathrm{S} 7+\mathrm{S} 8)$ ". Firstly, I want to explain why we did not describe any dissection of the N2 nodal stations as questioned by Dr. Hanna. We have described N2 nodal dissection in our previously published article (1). For this patient, we simply focused on sharing a step-by-step right $\mathrm{S} 7+8$ segmentectomy, and thus did not feel the need to describe N2 lymph node dissection. For pathologically confirmed lung cancer patients, we also start with a posterior mediastinal dissection in the same way that Dr. Hanna does.

Both Dr. Hanna and Dr. Kim shared with us a different 4-arm approach for the basal segmentectomy. At first, we also adopted the completely portal robotic lobectomy with the 4-arm (CPRL-4) technique, but with the accumulation of surgical experience, we gradually developed this new 3 -arm port placement strategy, with the bedside assistant playing a crucial part in the surgery. Compared to the port incision, the utility incision allows for more than one surgical instrument to pass through at the same time, so that all the dissecting, retracting, and transecting procedures can be done by the bedside assistant, which can facilitate the surgery. It is obvious that with fewer intercostal space (ICS) involved, patients might suffer less postoperative pain, but an extra port incision itself might also increase the pain. Therefore, which technique is less painful still needs to be clarified further.
Drs. Kim and Jeong have also done excellent work on robotic surgery. However, unlike their conclusions, our results confirm that 3 -port robotic assisted lobectomy (RAL3) is safer than video-assisted lobectomy (VAL) when considering its less intraoperative blood loss, shorter draining time, shorter postoperative length of stay, comparable operative time, and conversion and re-operation rate for early-stage NSCLC (1). The higher cost of robotic surgery is the main disadvantage compared to VATS. Our technique allows the assistant to expose the surgical field with one suction tip and one grasper, which is more flexible and time-saving than the CPRL-4 technique.

We are in complete agreement with that the new mapping techniques like 3D CT and ICG injection are necessary. Margin is the major concern when you choose to do a sublobar resection, while intersegmental plane identification is the key component for a segmentectomy. For lesions that do not situate nicely in a segment, we usually preoperatively localize the lesion with computed tomography-guided hook wire. However, this technique is invasive and unpleasant for patients. Obviously, the indocyanine green (ICG) near-infrared fluorescence localization technique is more minimally invasive and convenient, and could well complement the shortcomings of hook wire localization (2).

We highly appreciate the work done by Drs. Waël C. Hanna, Do Yeon Kim, and Jin Yong Jeong for their comments on our article. 


\section{Acknowledgments}

Funding: This work was supported by the Shanghai Hospital Development Center Grant (SHDC12016113).

\section{Footnote}

Conflicts of Interest: The authors have no conflicts of interest to declare.

Ethical Statement: The authors are accountable for all aspects of the work in ensuring that questions related to the accuracy or integrity of any part of the work are appropriately investigated and resolved.

\section{References}

1. Li JT, Liu PY, Huang J, et al. Perioperative outcomes of radical lobectomies using robotic-assisted thoracoscopic technique vs. video-assisted thoracoscopic technique: retrospective study of 1,075 consecutive p-stage I nonsmall cell lung cancer cases. J Thorac Dis 2019;11:882-91.

2. Mehta M, Sullivan K, Fahim C, et al. Near infrared imaging with indocyanine green mapping allows for extended margins in minimally invasive segmental resection. J Thorac Cardiovasc Surg 2018. In press.

Cite this article as: Li JT, Luo QQ. Reply to comments on "Robotic-assisted right medial and anterior basal segmentectomy (S7+S8)": lymph node dissection and incision. J Thorac Dis 2019;11(10):E192-E193. doi: 10.21037/jtd.2019.09.74 